\title{
Omics gets personal
}

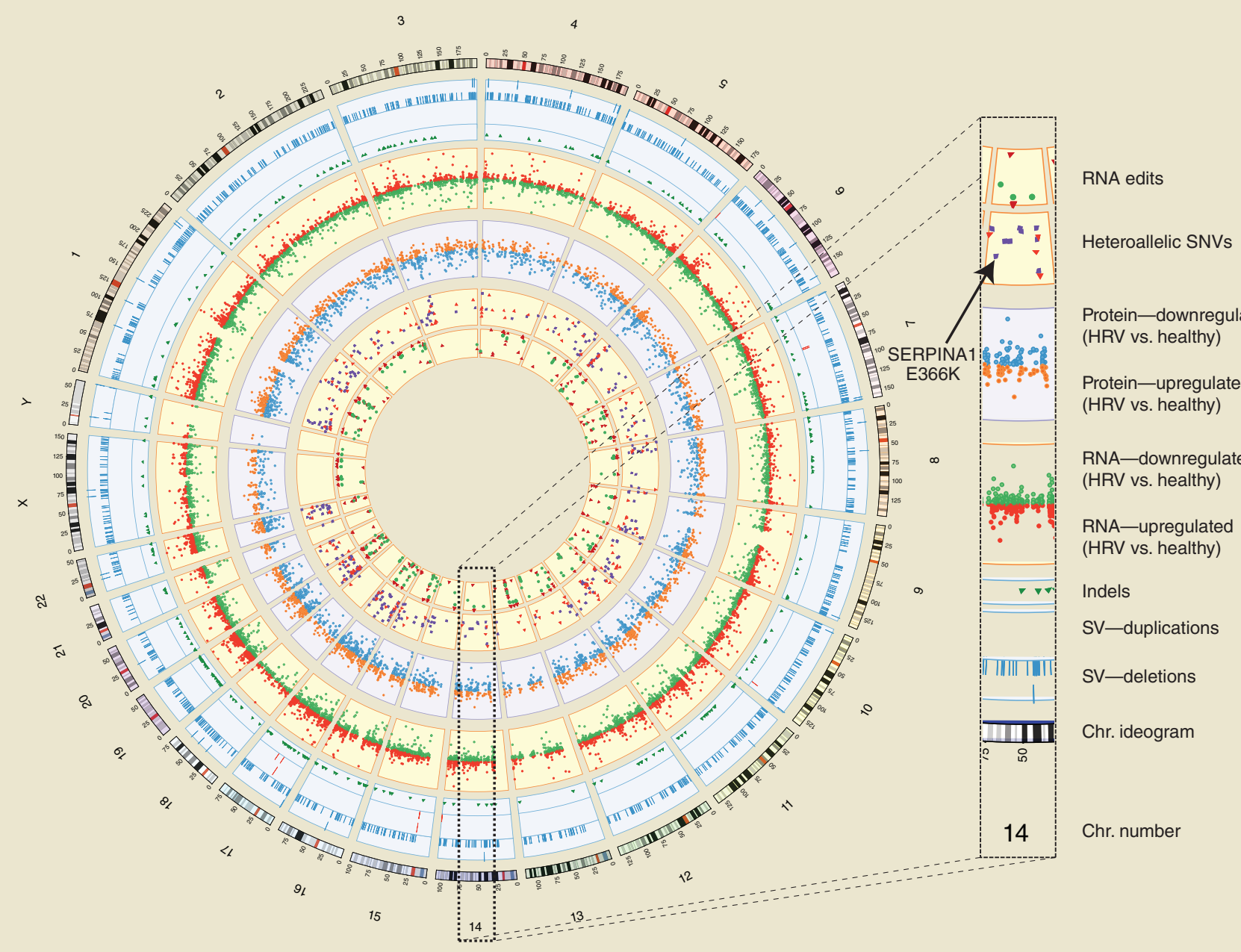

Figure 1 Schematic of iPOP. From outer to inner rings: chromosome ideogram; genomic data (pale blue ring) showing structural variants (SV) $>50$ bp (deletions, blue tiles; duplications, red tiles; indels, green triangles); transcriptomic data (yellow ring) showing ratio expression during human rhinovirus infection to that in healthy state; proteomic data (light purple ring) showing ratio of protein levels during HRV infection to healthy states; transcriptomic data (yellow ring) showing differential heteroallelic expression ratio of alternative allele to reference allele for missense and synonymous variants (purple dots) and candidate RNA missense and synonymous edits (red triangles, purple dots, orange triangles and green dots, respectively). Reprinted, with permission, from ref. 1.

The focus of omics tours de force has up to now been on complex diseases such as cancer or diabetes, with some successes. But now, Chen and colleagues ${ }^{1}$ show that combining profiling technologies to create an integrated personal omics profile (iPOP) for a generally healthy individual can provide a fascinating glimpse into a person's state of health as well as forecasting impending and-fortunately for the subject-sometimes actionable events. A key aspect of this study was its longitudinal nature, which involved frequent sampling for 14 months, intensified during two viral infections that the subject contracted during the study interval. Peripheral blood mononuclear cells, plasma and serum were collected and analyzed by deep whole-genome sequencing (with over 100-fold coverage), transcriptome profiling, proteome profiling (both targeted and not), metabolic profiling and autoantibody profiling, in conjunction with routine laboratory testing. Of several hundred thousand single-nucleotide variants (SNVs) and over 50,000 insertions or deletions (indels), a small number (51 and 4 , respectively) resulted in loss of gene function, and even fewer involved genes of known medical relevance. However, among those were genes associated with hypertriglyceridemia and type 2 diabetes (T2D). The risk for T2D proved to be real, as markers associated with diabetes (glucose and hemoglobinAlc levels) became elevated during the course of study, following a respiratory infection. Integrating data from the transcriptome, proteome and metabolome before and after viral infections allowed for the creation of a dynamic picture of the transition from health to infection and of the onset of T2D. Revealed was the coordinate expression of pathways after infectionsome expected, such as the upregulation of immune response pathways, and some not, such as the downregulation of the insulin signaling response pathway. This study illustrates that genomic data can direct the monitoring of specific diseases and shows how, by integrating omics, a detailed picture of disease states can be obtained.

Laura DeFrancesco

1. Chen, R. et al. Cell 148, 1293-1307 (2012). 